\title{
Beneficial effect of gelatin on iron gall ink corrosion
}

\author{
Alice Gimat $^{1 *} \mathbb{0}$, Anne Michelin¹, Pascale Massiani² and Véronique Rouchon ${ }^{1}$
}

\begin{abstract}
Iron gall Inks corrosion causes paper degradation (browning, embrittlement) and treatments were developed to tackle this issue. They often include resizing with gelatin to reinforce the paper and its cellulosic fibers (of diameter approx. $10 \mu \mathrm{m}$ ). This work aimed at measuring the distribution of ink components at the scale of individual paper fibers so as to give a better understanding of the impact of gelatin (re-)sizing on iron gall ink corrosion. For this purpose, scanning transmission X-ray microscopy (STXM) was used at the Canadian light source synchrotron (CLS, Saskatoon). This technique combines nano-scale mapping (resolution of $30 \mathrm{~nm}$ ) and near edge $X$-ray absorption fine structure (NEXAFS) analysis. Fe L-edge measurements enabled to map iron distribution and to locate iron(II) and iron(III) rich areas. N K-edge measurement made it possible to map gelatin distribution. C K-edge measurements allowed mapping and discrimination of cellulose, gallic acid, iron gall ink precipitate and gelatin. Three fibers were studied: an inked fiber with no size, a sized fiber that was afterwards inked and an inked fiber sprayed with gelatin. Analysis of gelatin and ink ingredients distribution indicated a lower amount of iron inside the treated cellulosic fiber, which may explain the beneficial effect of gelatin on iron gall ink corrosion.
\end{abstract}

Keywords: Gelatin, Iron-gallate ink, Paper, Sizing, STXM imaging

\section{Introduction}

Iron gall inks were widely in use for writing up to the nineteenth century. They are mainly composed of three ingredients: gallnuts extracts rich in phenolic compounds (gallic acid and polygalloyl esters of glucose such as pentagalloyl glucose or hexagalloyl glucose), an iron salt (often an iron sulfate, also called Vitriol) and a binder (usually a plant gum, such as gum Arabic) [1-3]. These inks, which contains both acids and iron, can induce cellulose depolymerization through two potential pathways: acid catalyzed hydrolysis [4-6] and radical oxidation, involving Fenton reaction and hydroxyl radicals [1].

The degradation of paper by iron gall inks, known as "iron gall ink corrosion", raises challenging conservation issues for drawings and archival records. From

\footnotetext{
*Correspondence: alice.gimat@mnhn.fr

${ }^{1}$ Centre de Recherche sur la Conservation des Collections (CRC, CNRS USR 3224), Sorbonne Université, Muséum National d'Histoire Naturelle, 36 rue Geoffroy St Hilaire, 75005 Paris, France

Full list of author information is available at the end of the article
}

the end of the twentieth century, conservation treatments were researched to prevent potentially both degradation pathways [7-11]. Identifying the dominant degradation pathway occurring on untreated documents is also a point of concern that remains difficult to scope in view of the shear variety of iron gall inks, papers and writing tools. Recent findings however suggest hydrolysis plays the most important role and that pure oxidative degradation induced by iron ions is not the main driving mechanism $[4-6,12]$. Three main facts point to this hypothesis. Firstly, no hydroxyl radicals could be detected with trapping techniques below $\mathrm{pH} 6$ probably because they recombine with available protons in this $\mathrm{pH}$ range [4]. Secondly, the degradation of paper by iron-gallate ink has an activation energy of $95-98 \mathrm{~kJ} \cdot \mathrm{mol}^{-1}$ [6], a value that is compatible with acid-catalyzed cellulose hydrolysis and much higher than those of cellulose oxidative mechanism. Thirdly, the presence of iron in the inks participates to acid-catalyzed depolymerization: on cellobiose, it was

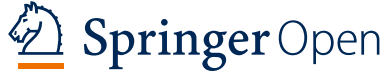

(c) The Author(s) 2021. Open Access This article is licensed under a Creative Commons Attribution 4.0 International License, which permits use, sharing, adaptation, distribution and reproduction in any medium or format, as long as you give appropriate credit to the original author(s) and the source, provide a link to the Creative Commons licence, and indicate if changes were made. The images or other third party material in this article are included in the article's Creative Commons licence, unless indicated otherwise in a credit line to the material. If material is not included in the article's Creative Commons licence and your intended use is not permitted by statutory regulation or exceeds the permitted use, you will need to obtain permission directly from the copyright holder. To view a copy of this licence, visit http://creativecommons.org/licenses/by/4.0/. The Creative Commons Public Domain Dedication waiver (http://creativeco mmons.org/publicdomain/zero/1.0/) applies to the data made available in this article, unless otherwise stated in a credit line to the data. 
demonstrated that the oxidation of iron(II) to iron(III), led to the acidification of the medium and was responsible for the hydrolysis of the glycosidic bond [5]. For this reason, the presence of free iron(II) is considered to be detrimental to paper conservation.

Yet, the degradation of paper by iron gall ink is governed not only by chemical reactions, but also by the physical penetration of ink (and iron) within the paper. Paper fibers come from the paper pulp and are initially highly permeable to water. During the paper making process, sheets are impregnated with a size that makes them less permeable to water and thus suitable for writing [13]. The size is also a key parameter to prevent the migration of ink on the verso of the paper sheet. In case of reactive inks, such as iron gall inks, it also limits future chemical interactions between the ink components and the paper support.

The penetration depth of modern inks into paper sheets has been studied with several techniques, such as optical microscopy [14-16], confocal laser scanning microscopy [17], scanning electron microscopy (SEM) [18], X-ray microtomography and laser ablation [19]. Particle-induced X-ray emission (PIXE) has also been used to measure the penetration of metallic ink components $(\mathrm{Fe}, \mathrm{Zn}, \mathrm{Cu}$ ) into unsized paper cross sections with a spatial resolution of $1.5 \mu \mathrm{m}^{2}$ [20]. Yet, water transport in paper sheets (usually about $70-100 \mu \mathrm{m}$ thick) does not only involve pores and capillaries between fibers but also the fibers themselves, the diameter of which is of the order of $10 \mu \mathrm{m}$ [21]. Investigating the migration of ink components within paper fibers thus requires analytical tools capable of mapping components distributions within a paper fiber, i.e., at the nanoscale.

Before the industrial revolution, papers were sized with gelatin [22, 23], which coats the paper and also penetrates into the sheets $[13,24]$. Yet, it has been shown recently that gelatin was not penetrating the paper fibers [25]. Yet gelatin that bears electronegative groups (such as carboxylates) can interact chemically with cations such as iron [26]. It can also be affected by the ink since it has been shown that the paper of original manuscripts is often more permeable to water on the verso of the ink line than on blank areas [11]. This observation corresponds to an alteration of the size by iron gall ink, which is not yet completely understood. In addition, gelatin is currently used in paper conservation workshops for mending or for re-sizing fragile papers (or papers that have undergone washing) [27]. Moreover, it has been shown that a certain type of gelatin, used as resizing agent, induced a decrease of iron gall ink corrosion at the macroscopic and macromolecular scale [28, 29], thus highlighting interactions occurring between gelatin and the paper/iron gall ink system.
Studying migration of gelatin and ink components into paper fibers will help understanding the beneficial effect of gelatin on iron gall ink corrosion. Given the diameter of paper fibers (approx. $10 \mu \mathrm{m}$ ), scanning transmission X-ray microscopy (STXM) offers promising perspectives. It requires synchrotron facilities but combines (i) chemical speciation-sensitive images at a spatial resolution down to $30 \mathrm{~nm}$ and (ii) X-ray Absorption measurements (with a spectral resolution of $0.1 \mathrm{eV}$ ), referred to as near edge X-ray absorption fine structure (NEXAFS) or X-ray absorption near edge structure (XANES). Although NEXAFS spectroscopy (at the Fe K-edge) has already been largely used on iron gall inks to quantify iron(II) to iron(III) ratios in both historical and model iron-gallate inks [30-33], imaging was not carried out. Preliminary measurements on inked paper fibers clearly established the interest of the STXM to map ink components at the fiber scale using the Fe L- and C K-edges [34]. Based on this first work, the present study uses the STXM technique to investigate gelatin and iron-gallate ink distribution inside individual paper fibers. It aims at a better understanding of the impact of gelatin on iron gall ink corrosion.

\section{Results and discussion}

For the study, a linen rag paper pulp, prepared by stamper beating at Moulin du Verger (Charentes, France) was chosen to model historical papers. It contains no size and no fillers. Fibers were extracted and prepared as described in Material and Methods to obtain three samples: I; G_I and I_G. I is an inked fiber supposed to model the extreme case of an un-sized paper in contact with a drop of ink; G_I is a sized and inked fiber, supposed to model sized paper coated with iron-gallate ink and I_G is an inked fiber treated with gelatin, supposed to model a poorly sized paper (no size) coated with ink, and spayed in a conservation workshop with a gelatin solution. Note that this is a preliminary work as only three samples could be analysed during the synchrotron shift. Historical fibers were not considered in this study, but it would be interesting to compare the iron distribution inside historical papers and their conservation state, on archival documents that can be sampled. Thin-sections of the fibers were obtained by Focussed Ion Beam, positioned on a labelled copper disc (see "Material and methods" Section; Additional file 1: Figure S1) and were imaged in situ with scanning electron microscopy (SEM). Then, STXM imaging was carried out at the Canadian light source facility (CLS, Saskatoon, Canada) on the SM 10ID-1 Beamline which provides soft X-rays $(130-2700 \mathrm{eV})[35,36]$. This technique allows to have a map of the X-ray absorption at several energies and a NEXAFS spectrum for each point of the STXM image (Additional file 1: Figure S2). The 

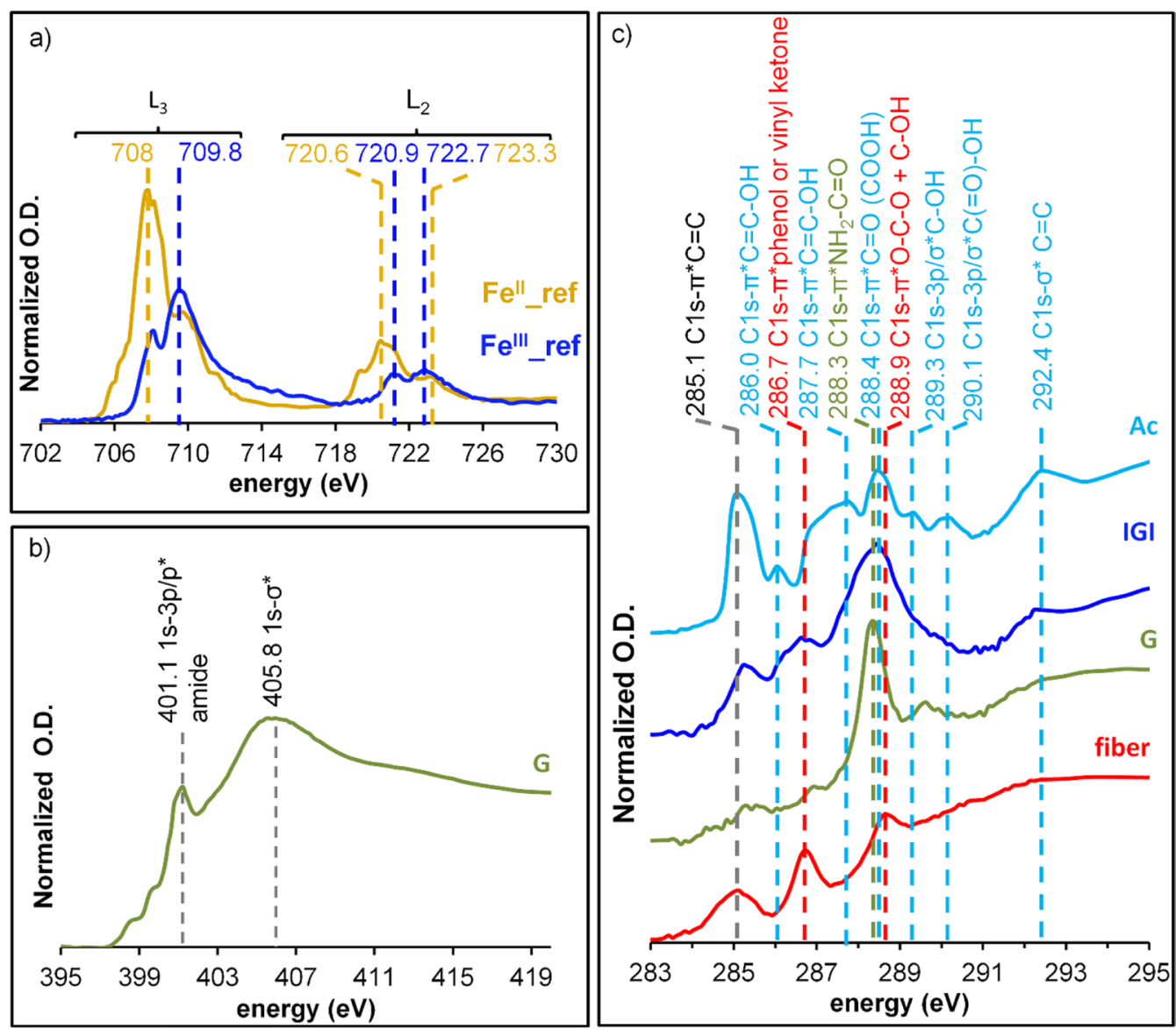

Fig. 1 NEXAFS spectra of reference compounds at the three edges studied. a Reference of iron(II) chloride (Fe'_ref) and iron(III) chloride (Fe'III_ref) at the Fe L-edge; b gelatin (G) at the N K-edge and c C K-edge of gallic acid reference (Ac), iron-gallate ink precipitate (IGI), gelatin (G) and raw linen rag fiber (fiber). Attribution was based on several previous studies [34, 37-41]

measurements were done at the Fe L-edge (700-720 eV), C K-edge (270-325 eV) and N K-edge (395-435 eV) (see "Material and methods" Section; Additional file 1: Figure S2) to monitor sample absorption and the distribution of organic compounds inside the cross-sections. Note that in all figures, maps have been subtracted with map of the same location taken at an energy before the edge (i.e., $700 \mathrm{eV}$ for $\mathrm{Fe}, 398 \mathrm{eV}$ for $\mathrm{N}$ and $280 \mathrm{eV}$ for $\mathrm{C}$ ) unless otherwise stated. This allows a better visualization of element distribution.

\section{Reference NEXAFS signatures and components maps}

Collecting NEXAFS spectra of reference compounds at the Fe L-edge, C K-edge and N K-edge (Fig. 1) was necessary for comparison with those extracted from fiber samples data stacks. They were also used as reference spectra for mapping the distribution of individual compounds in the samples and for obtaining RGB maps.

At the Fe L-edge, the references $\mathrm{Fe}^{\mathrm{II}}$ _ref and $\mathrm{Fe}^{\mathrm{III}}{ }_{-}$ref present four main peaks at $708 \mathrm{eV}$ (Fe L3 edge), $709.8 \mathrm{eV}$
(Fe L3 edge), near 720-721 eV (Fe L2 edge) and near $723 \mathrm{eV}$ (Fe L2 edge) (Fig. 1a) [37, 38]. The peaks intensities of these two references are different which, in principle, makes it possible to quantify the $\mathrm{Fe}^{\mathrm{II}}-\mathrm{Fe}^{\mathrm{III}}$ ratio as was done on silicates systems [42]. However, here, no precise quantification was carried out as the reduction of iron under the beam could not be neglected (optical density changes up to $15 \%$ were measured during an acquisition). These Fe L-edge spectra also helped choosing STXM energies that are appropriate to map $\mathrm{Fe}^{\mathrm{II}}$ or $\mathrm{Fe}^{\mathrm{III}}$ rich regions (Figs. 2, 3, 4): they show that $708 \mathrm{eV}$ is preferentially related to $\mathrm{Fe}^{\mathrm{II}}$ while $709.8 \mathrm{eV}$ is more specific of $\mathrm{Fe}^{\mathrm{III}}$. In order to enhance the readability of the images, the $700 \mathrm{eV}$ maps (before the edge) were subtracted from the $708 \mathrm{eV}$ maps (characteristic of $\mathrm{Fe}^{\mathrm{II}}$ ). Similarly, the $708 \mathrm{eV}$ maps (specific of $\mathrm{Fe}^{\mathrm{II}}$ ) were subtracted from the $709.8 \mathrm{eV}$ maps (specific of $\mathrm{Fe}^{\mathrm{III}}$ ). These subtractions gave a more contrasted view of respectively $\mathrm{Fe}^{\mathrm{II}}$ and $\mathrm{Fe}^{\mathrm{III}}$ distributions. 

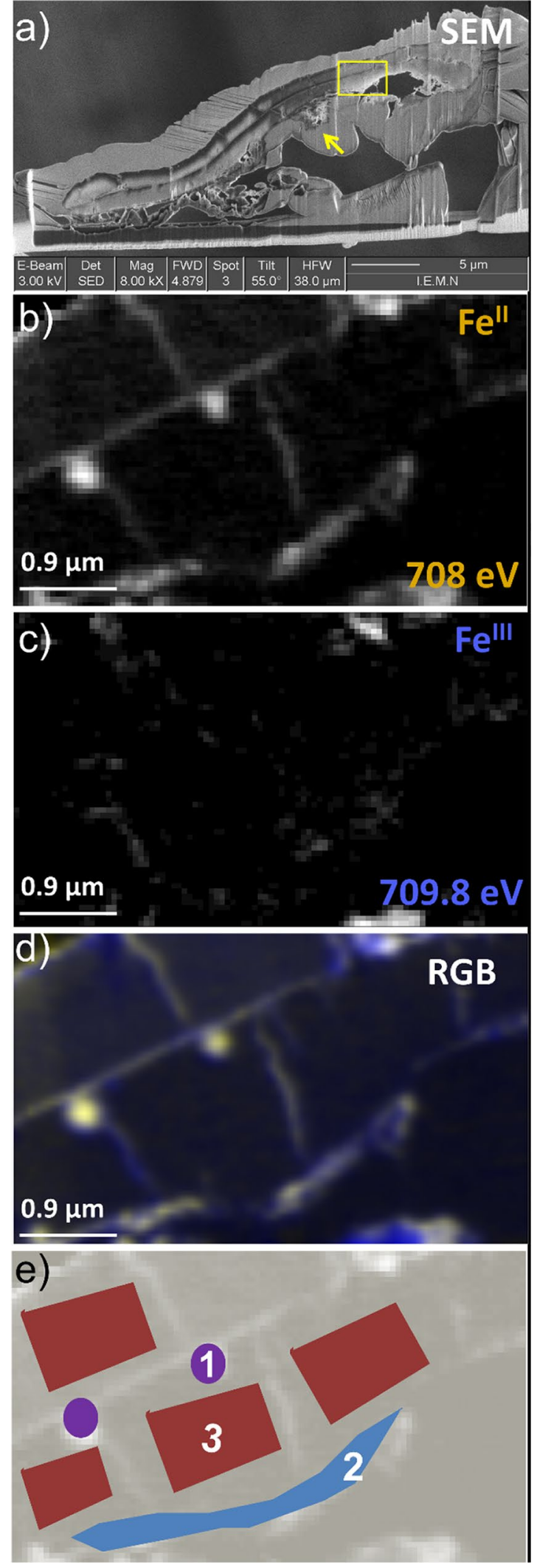

4 Fig. 2 Mapping of the inked fiber (I). a SEM image of the thin section; b and c STXM maps of a restricted area (yellow square) at the Fe L-edge: $\mathbf{b}$ the map at $708 \mathrm{eV}$ (after subtraction of maps at $700 \mathrm{eV}$ ) represents Fe" distributions; $\mathbf{c}$ the map at $709.8 \mathrm{eV}$ (after subtraction of maps at $708 \mathrm{eV}$ ) represents Fe $\mathrm{e}^{\text {III }}$ distributions; $\mathbf{d}$ RGB picture resulting from fit of the stack with Fe" (Fig. 1a, yellow) and Fe IIII (Fig. 1a, blue) reference spectra. e Regions of interest (from numbers 1 to 3 ) chosen to extract the NEXAFS spectra shown on Fig. 3a

Gelatin is the only compound of the system that contains nitrogen. Hence, $\mathrm{N}$ K-edge mapping will be specific of the presence of gelatin. At this edge, the NEXAFS spectrum of gelatin (Fig. 1b) presents a broad band at $405.7 \mathrm{eV}$ that corresponds to $1 \mathrm{~s}-\sigma^{*}$ transition [41], and a main peak at $401.1 \mathrm{eV}$ that corresponds to amide groups. The distribution of gelatin was also obtained with maps at $401.1 \mathrm{eV}$ from which maps at $398 \mathrm{eV}$ (before the edge) were subtracted in order to enhance the contrast.

At the $\mathrm{C}$ K-edge, the raw linen rag fiber NEXAFS spectrum (Fig. 1c, fiber) is similar to previously reported cellulosic fibers [34, 39]. It shows three peaks corresponding to aromatic carbons $(285.1 \mathrm{eV})$ and vinyl ketone (a ketone conjugated with a double bond) $(286.7 \mathrm{eV})$, possibly formed during milling, and $\mathrm{C}-\mathrm{O}$ transition of glycosidic bonds and alcohol groups $(288.9 \mathrm{eV})$. The gallic acid spectrum (Fig. 1c, Ac) also matches previously published data $[34,40]$ with eight peaks corresponding to $1 \mathrm{~s}-\pi^{*}$ transitions of aromatic carbons $(285.1 \mathrm{eV})$, aromatic alcohol (286.0-288 eV), carboxylic group (288.4 eV), $1 \mathrm{~s}-3 \mathrm{p} / \sigma^{*}$ transition of alcohol $(289.3 \mathrm{eV}$ and $290.1 \mathrm{eV})$ and $1 \mathrm{~s}-\sigma^{*}$ carbon transition $(292.4 \mathrm{eV})$.

The spectrum of the IGI precipitate (Fig. 1c, IGI) presents some common feature with the one of gallic acid with the signature of aromatic carbon $(\mathrm{C}=\mathrm{C}, 285.1 \mathrm{eV})$, aromatic carbon connected to hydroxyl group $(\mathrm{C}=\mathrm{C}-$ $\mathrm{OH}, 286-287 \mathrm{eV})$, carboxylic groups $(288.4 \mathrm{eV})$ and $1 \mathrm{~s}-\sigma^{*}$ carbon transition $(292.4 \mathrm{eV})$. However, for the IGI sample, the peak at $288.4 \mathrm{eV}$ is enhanced in comparison to the other peaks and larger than for gallic acid. This suggests modifications in the environment of the carboxylic acid groups which is coherent with a precipitate formation (iron chelation in precipitate is done via phenolic hydroxyls groups and the carboxylic acid group [43]. Differentiating gallic acid and iron-gallate precipitate in a mixture should be possible due to their distinct spectra and respective peaks intensities.

The gelatin spectrum (Fig. 1c, G) exhibits a main peak at $288.3 \mathrm{eV}$ related to the amide groups. Some other minor contributions are observed at $285.5 \mathrm{eV}, 287 \mathrm{eV}$ and $289.5 \mathrm{eV}$, but these signatures are too weak to be used for discrimination.

These preliminary observations show that cellulose, gelatin and ink components have different $\mathrm{C}$ K-edge 

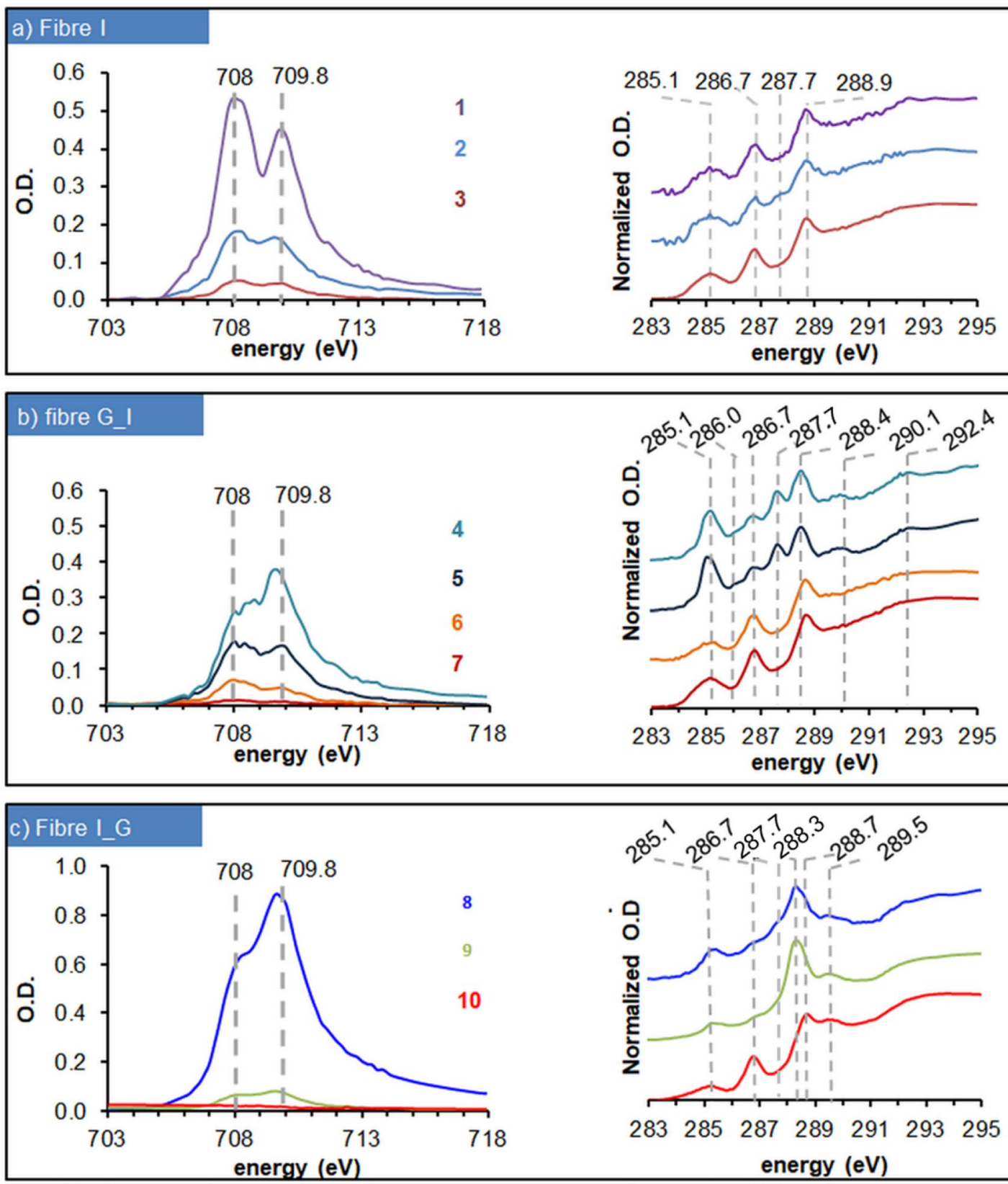

Fig. 3 NEXAFS spectra extracted from the regions of interest of the unsized and inked fiber I (a), sized and inked fiber G_I (b) and gelatin sprayed inked fiber I_G (c). The spectra correspond to the Fe L-edge (left) and C K-edge (right). They were extracted from the stacks in the region of interest 1-10 represented in Figs. 2, 4, 5. C K-edge spectra were normalised with the Athena software. Fe L-edge spectra are presented un-normalized to better assess the relative amount of iron present in the thin section

NEXAFS signatures. Fixed energies were also used to quickly map the distribution of gallic acid $(287.7 \mathrm{eV})$, cellulose $(286.7 \mathrm{eV})$, gelatin $(288.3 \mathrm{eV})$ and IGI $(285.2 \mathrm{eV})$ (with subtraction of maps before the C K-Edge). Yet, as compounds signatures partially overlap, the absorption contrast maps at a single energy were crosschecked carefully with extracted spectra taken from the regions that were supposed to be rich in the spotted component. Comparing maps at fixed energy and NEXAFS spectra obtained at other edges ( $\mathrm{Fe} \mathrm{L}$ and $\mathrm{N} \mathrm{K}$ ) also appeared useful to study properly the distribution of these different components. 


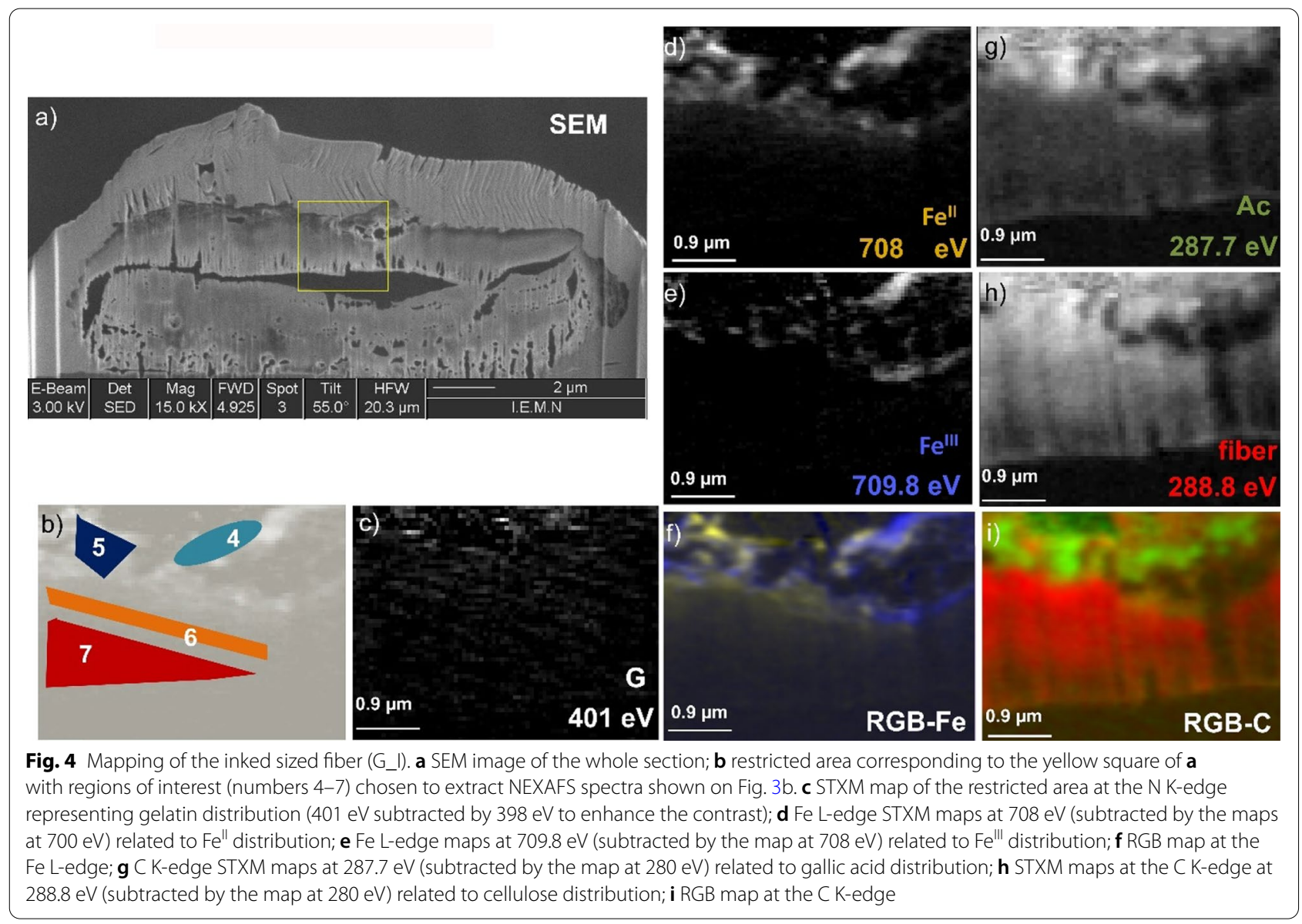

\section{Analysis of unsized fiber I: iron absorption in the fiber}

With SEM imaging (Fig. 2a), it can be seen that the fiber I presents an unusual structure with no lumen visible. Instead, the fiber seems very large (approx. $30 \mu \mathrm{m}$ ) and extremely flat (Thickness approx. $4 \mu \mathrm{m}$ ). This unexpected structure suggests that the original fiber was opened lengthwise during the stamper beating. The two walls would then correspond to the inner and outer walls of the former fiber.

Fe L-edge maps (Additional file 1: Figure S3) show a significant presence of iron around the fiber, consistent with a thin ink layer. Outside the fiber, mainly one region is brighter on the map at $709.8 \mathrm{eV}$ (Additional file 1: Figure $\mathrm{S} 3$, yellow arrow), suggesting aggregates rich in $\mathrm{Fe}^{\mathrm{III}}$ probably corresponding to IGI precipitate. Inside the fiber, iron seems to concentrate along cracks and mainly correspond to $\mathrm{Fe}^{\mathrm{II}}$ as it is particularly noticeable on the map at $708 \mathrm{eV}$. On an enlarged region of the fiber I (Fig. 2b, c), stack fits were performed using the Axis2000 software to get a RGB map (Fig. 2d) showing the distribution of $\mathrm{Fe}^{\mathrm{II}}$ (in yellow) and $\mathrm{Fe}^{\mathrm{III}}$ (in blue) based on a comparison with the reference compounds spectra (Fig. 1a). The surface of the fiber is coated relatively homogeneously with a thin $\mathrm{Fe}^{\mathrm{II}}$ rich layer that also follows cracks in the inner part of the fiber. Many of these cracks are oriented perpendicular to the walls, and were probably formed during paper milling. Bright Fe-rich spots (Fig. 2e, number 1), approx. $200 \mathrm{~nm}$ wide are also observed along the cracks and are predominantly composed of $\mathrm{Fe}^{\mathrm{II}}$.

Extraction of Fe L-edge spectra from the regions of interest 1-3 (Figs. 2e, 3a, left), showed that iron is not only distributed along cracks but also penetrates in the fiber (region 3) where it is present at low concentration (low O.D.) (Fig. 3a, left, spectrum 3). Although the spectra 1-3 significantly differ in optical density, they have a similar shape, the peak at $708 \mathrm{eV}$ being more intense than the peak at 709.8. This feature corresponds to a $\mathrm{Fe}^{\mathrm{II}} /$ $\mathrm{Fe}^{\mathrm{III}}$ mixture with a predominance of $\mathrm{Fe}^{\mathrm{II}}$ versus $\mathrm{Fe}^{\mathrm{III}}$. $\mathrm{C}$ K-edge spectra were also extracted from these three regions of interest (Fig. 3a, right). They were all similar to the spectrum of cellulose recorded on the raw fiber (Fig. 1c, spectrum fiber). This is consistent with the 
fact that, even if the fiber is inked, cellulose remains its main organic constituent. On the spectrum extracted from region 2, at the surface of the fiber (Fig. 3a, right, spectrum 2), there is a shoulder at $287.7 \mathrm{eV}$ that probably comes from gallic acid or IGI. This signature is not perceptible in the inner part of the fiber (regions 1 and 3, Fig. 3a, right, spectra 1 and 3), suggesting that gallic acid and IGI do not migrate in significant amount within the fiber and mostly remain outside. As no other carbon signature than cellulose was detected inside the fiber (region 1, Fig. 3a, left, spectrum 1), the iron that has migrated inside the fiber might be chelated to sulfates or to cellulose hydroxyl groups. Iron(II) presence inside the fiber center was also observed in the preliminary STXM study carried out on bleached fibers [34]. The fact that iron is mainly present as iron(II) inside the fiber helps understanding why unsized papers that are impregnated by iron gall ink can be altered within a few months [33]. Indeed, protons can be released during the formation of iron(III) oxyhydroxides during Fe(II) oxidation $^{1}$ or iron-gallate ink complex from iron-gallic acid binding $[43,44]$, thus lowering the local $\mathrm{pH}$ and promoting acid hydrolysis of cellulose macromolecules. As polymeric chains are getting shorter, paper gradually loses its mechanical properties.

\section{Analysis of fiber G_I: gelatin prevents iron migration}

The fiber G_I presents a rather classical linen rag fiber shape (ovoid) with a central hole corresponding to the lumen (Fig. 4a). The SEM image shows some damage due to the milling process (holes where the FIB section is too thin).

Fe L-edge maps (Additional file 1: Figure S4) highlight regions containing iron that are mostly located outside the fiber, suggesting some coating with an ink layer. The Fe L-edge mapping (Fig. 4d, e) confirmed that iron remains mainly outside the fiber with an uneven $\mathrm{Fe}^{\mathrm{II}}$ and $\mathrm{Fe}^{\mathrm{III}}$ distribution.

Only small amounts of iron (mostly $\mathrm{Fe}^{\mathrm{II}}$ ) penetrate in the fiber as attested by NEXAFS iron spectra (Fig. 3b, left, spectra 6 and 7) and the RGB iron map (Fig. 4f). Moreover, the concentration in iron decreases with the distance to the surface, since the sub surface (Fig. 4f, region 6; Fig. 3b, left, spectrum 6) contains greater amounts of iron than the inner part of the fiber (Fig. 4f, region 7; Fig. 3b, left, spectrum 7).

The C K-edge NEXAFS spectra obtained inside the fiber (Fig. 4b, regions 6 and 7; Fig. 3b, right, spectra 6 and 7) match the fiber reference with characteristic peaks at 285.1, 286.7 and $288.9 \mathrm{eV}$ (Fig. 1c, spectrum fiber). In contrast, the $\mathrm{C}$ K-edge NEXAFS spectra of regions

${ }^{1}$ Global equation:4Fe ${ }^{2+}+\mathrm{O}_{2}+6 \mathrm{H}_{2} \mathrm{O} \rightarrow 4 \mathrm{FeOOH}+8 \mathrm{H}^{+}$. located outside the fiber (Fig. 4b, regions 4 and 5; Fig. 3b, right, spectra 4 and 5) attest of the presence of gallic acid with characteristic signatures at $286.0 \mathrm{eV}, 286.5 \mathrm{eV}$, $287.7 \mathrm{eV}$ and $288.4 \mathrm{eV}$. Yet some contribution from IGI cannot be excluded as it also presents two characteristic peaks at $286.7 \mathrm{eV}$ and $288.4 \mathrm{eV}$ that superimpose those of gallic acid (Fig. 1c).

The C K-Edge characteristic peak of gelatin at $288.3 \mathrm{eV}$ (Fig. 1c) is too close from the above-mentioned peaks to allow detection of gelatin in the C K-Edge data without ambiguity. That is why N K-Edge was also used. Previous work performed on similar fibers, sized in a similar way (yet without IGI application) showed that gelatin does not penetrate the paper fiber, but simply coats it [25]. These measurements were performed with the same system using similar acquisition parameters than in the present study, and $\mathrm{N}$ K-edge maps allowed an easy location of gelatin. On the present fiber G_I however, the N K-edge maps at $401 \mathrm{eV}$ (an energy characteristic of gelatin amide bonds) are highly noisy (Fig. 4c), as well as the NEXAFS spectra taken outside the fibre, which show only a weak and broad signal (Additional file 1: Figure S5, spectra 4 and 5). This suggests that only traces of nitrogen, most probably gelatin, are present outside the fiber but at low concentration, close to the detection limit.

These examinations of STXM maps and NEXAFS spectra suggest that most of the gelatin formerly coating the fiber was released in the solution during ink impregnation. This removal was not expected since gelatin is known to be insoluble in water at ambient temperature. We suppose here that the presence of iron, sulfates and hydronium ions in the ink solution contributed to the partial solvation and dissolution of gelatin, due to a salting-in effect.

The C K-edge and Fe K-edge stacks evidence that a layer of ink (region with high iron content, containing gallic acid and possibly IGI) coats the cellulosic fiber and that only the fiber sub surface contains iron (mostly $\mathrm{Fe}^{\mathrm{II}}$ ), as illustrated by the RGB maps (Fig. 4f, i). On the iron RGB map (Fig. 4f), a small penetration of $\mathrm{Fe}^{\mathrm{II}}$ is seen on a depth of a few hundreds of nanometres. It corresponds to a maximum O.D. of 0.07 (Fig. 3b, left, spectrum 6), a value close to the value of 0.05 measured in the inner part of the unsized fiber I (Fig. 3a, left, spectrum 3). These two values are much higher than the maximum O.D. found in the inner part of the G_I fiber sample (Fig. 3b, left, spectrum 7 ), meaning that the former presence of gelatin significantly prevented migration of iron in the fiber.

\section{Analysis of fiber I_G: impact of a spray of gelatin on iron distribution}

Previous studies have shown that depositing gelatin on an inked paper can slow down the depolymerisation process 
[29]. Thus, applying gelatin on graphic documents during conservation treatments should limit iron gall ink corrosion. The I_G fiber was therefore prepared to investigate possible interactions between ink and gelatin when this latter is applied a posteriori.

In the SEM image of the thin section I_G, three fibers can be distinguished (Fig. 5a, A, B and C). Even if the lower part of the foil was damaged by the FIB milling, the upper part was appropriate for analysis. At the $\mathrm{N}$ K-edge, the presence of gelatin was clearly established by STXM mapping at $401 \mathrm{eV}$, an energy characteristic of amide bonds (Fig. 5h). As expected [25], gelatin coats the three fibers without migrating inside (Fig. 5h; Additional file 1: Figure S6). It is also noticeable inside the lumen of fiber $B$ probably because this fiber was initially opened on one end, allowing gelatin solution to migrate in the lumen along the fiber axis. At the C K-Edge, the distribution of gelatin can also be seen on the map at $288.3 \mathrm{eV}$ (Fig. 5i), an energy that is specific of the gelatin NEXAFS spectrum (Fig. 1c, spectrum G). On this map, the gelatin free areas correspond to the cellulosic fibers for which a good response is obtained at $286.7 \mathrm{eV}$ (C K-edge, Fig. 5e). As for the maps recorded at the Fe L-edges, they show that the gelatin coating contains some amount of iron distributed relatively evenly together with bright spots rich in iron (Additional file 1: Figure S6).

On the enlarged region of the map (Fig. 5, yellow square), complete stacks were recorded at the Fe L-and $\mathrm{C}$ K-edges and NEXAFS spectra were extracted from different regions: in the spots rich in iron (Fig. 5 j, region 8), in the gelatin (Fig. 5j, region 9) and in the fiber (Fig. 5j, region 10). Iron rich spots (region 8) mostly correspond to $\mathrm{Fe}^{\mathrm{III}}$ (Fig. 3c, left, spectrum 8). On these spots, the C K-edge signature (Fig. 3c, right, spectrum 8 ) is close to the spectrum of the IGI precipitate, with characteristic peaks at $285.1 \mathrm{eV}, 286.7 \mathrm{eV}$ and $288.4 \mathrm{eV}$ (Fig. 1c). Yet the most intense peak at $288.4 \mathrm{eV}$ also appears slightly asymmetric, with a sharp top at $288.3 \mathrm{eV}$ that is interpreted as a contribution of gelatin to the signal. This point is confirmed by the fact that this region appears relatively bright on the N K-Edge map at $401 \mathrm{eV}$. An additional contribution of gallic acid in the C K-edge spectrum of region 8 is seen in the shoulder observable at $287.7 \mathrm{eV}$. These observations led to conclude that region 8 is rich in iron-gallate ink (consistent with the high content in $\mathrm{Fe}^{\mathrm{III}}$ ) but also contains some minor proportion of gelatin and gallic acid. Outside the fiber, in the gelatin coating (Fig. 5j, region 9), some amount of iron is detected, mostly as $\mathrm{Fe}^{\mathrm{III}}$ (Fig. 3c, left, spectrum 9). The C K-Edge signature of this region (Fig. 3c, right, spectrum 9) is similar to the one of gelatin (Fig. 1c, spectrum G), meaning that no other organic component is detected in this region.

Conversely, in the inner part of the fiber (Fig. 5j, region 10), the C K-Edge signature is similar to the one of cellulose (Fig. 3c, right, spectrum 10) with an additional small and broad peak at $289.5 \mathrm{eV}$. This peak was previously observed in the secondary wall of a linen rag fiber and also on some cellulosic reference [34] and is probably due to $\mathrm{C}-\mathrm{OH}$ bonds.

A stack fit treatment of the data was performed at the $\mathrm{C}$ K-edge with the model spectra of the raw fiber, gelatin and IGI precipitate (Fig. 1c). The resulting RGB map (Fig. 5g, RGB map) illustrates that gelatin remains outside the fiber and contains some grains of IGI precipitate.

Interestingly, no iron was detected in region 10, inside the fiber (Fig. 2c, left, spectrum 10). This point was completely unexpected since small concentrations of iron were detected inside the two previously analysed fibers (I and G_I). Before impregnation with gelatin, the fiber I_G was expected to be comparable to fiber I and thus probably containing similar amount of iron (mainly as $\mathrm{Fe}^{\mathrm{II}}$ ). Consequently, the fact that no trace of iron could be detected on the I_G fiber suggests that $\mathrm{Fe}^{\mathrm{II}}$ was removed from the inner part of the fiber during the gelatin spray. This is consistent with the fact that iron is found in the gelatin coating (Fig. 2c, left, spectrum 10). It is indeed of common knowledge that iron that is not involved in the IGI precipitate (such as $\mathrm{Fe}^{\mathrm{II}}$ ) is highly water soluble [45, 46]. When the paper is humidified, it can easily migrate out of the ink line [11]. During the gelatin spray, gelatin coats the fiber without migrating inside, but water goes in the fiber, thus allowing the dissolution of iron and its migration out of the fiber in the gelatin solution. Then the solution cools down rapidly, forming a gel that fixes iron. The high predominance of $\mathrm{Fe}^{\mathrm{III}}$ in gelatin suggests oxidation reactions occurring with gelatin consistent with the ability of gelatin to chelate iron [28, 47-49]. These chelation mechanisms may also favour iron migration by entropic effect thus contributing to the decrease of iron concentration in the inner part of

(See figure on next page.)

Fig. 5 Mapping of the inked fiber sprayed with gelatin (I_G). a SEM image of the whole section showing three fibers (A-C). b and c STXM maps of the restricted area (yellow square) at Fe-K edge. b Fe K-edge maps at $708 \mathrm{eV}$ (subtracted by maps at $700 \mathrm{eV}$ ) related to Fe" distribution. c Fe K-edge maps at $709.8 \mathrm{eV}$ (subtracted by maps at $708 \mathrm{eV}$ ) related to Fe"l distribution. $\mathbf{d}$ RGB picture resulting from fit of the $\mathrm{F}$ L-edge stack with Fe" (yellow) and Fe"l' (blue) reference spectra of Fig. 1a. e, f, i C K-edge maps at $286.7 \mathrm{eV}, 285.2 \mathrm{eV}$ and $288.3 \mathrm{eV}$ (from which maps at $280 \mathrm{eV}$ were subtracted) showing respectively the location of the cellulose fiber, IGI precipitate and gelatin. $\mathbf{g}$ RGB picture resulting from fit of the C K-edge stack with fiber (red), IGI (blue) and gelatin (green) reference spectra of Fig. 1a. h N K-edge map at $401 \mathrm{eV}$ (subtracted by maps at $398 \mathrm{eV}$ ) highlighting gelatin distribution. j Regions of interest (numbers 8-10) chosen to extract the NEXAFS spectra of Fig. 3C 

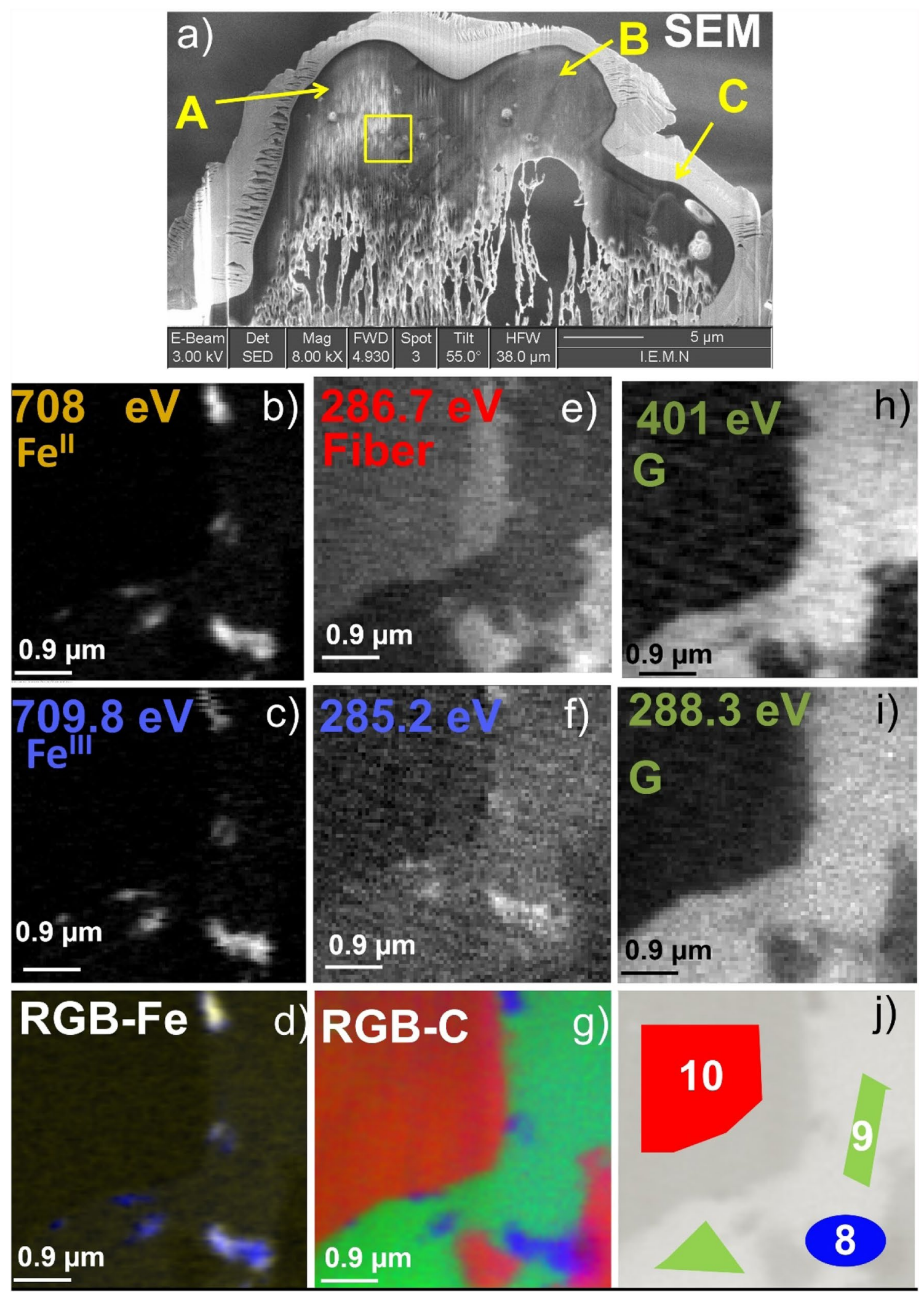

Fig. 5 (See legend on previous page.) 
the fiber below the limit of detection. Iron trapping by gelatin would explain the lower depolymerization of cellulose observed in presence of gelatin on iron-gallate inked papers [29]. Indeed, as iron enhances cellulose degradation [5, 6], its removal from the inner part of the fiber contributes to limit its interaction with cellulose and thus the cellulose decay.

\section{Conclusion}

The aim of this study was to get a better understanding of the impact of gelatin on iron gall ink corrosion through the study at a nano-scale of the distribution of gelatin and iron-gallate ink in individual paper fibers. FIB milling was used to prepare thin slices from inked and/or gelatin sized fibers. On these slices, the distribution of gelatin and ink components (including gallic acid, iron and iron-gallate ink precipitate) were then mapped by STXM at the Fe L-, $\mathrm{C} \mathrm{K}$ - and $\mathrm{N}$ K-edges.

On a fiber that was inked but not sized, the ink mostly formed an outside layer including some iron clusters. The exact nature of these clusters could not be assessed because they did not show a specific C K-edge signature. They might correspond to iron sulfate. More interestingly, some iron (most probably free $\mathrm{Fe}^{\mathrm{II}}$ ) was able to penetrate in the fiber. It was homogeneously distributed at low concentration inside the fiber, but spots of high concentration in iron were also found along cracks. The presence of $\mathrm{Fe}^{\mathrm{II}}$ inside the fiber can be seen as a factor favouring low $\mathrm{pH}$ values inside the fiber, thus promoting acid hydrolysis of cellulose macromolecules. Indeed, protons can be released during the formation of iron(III) oxyhydroxides during $\mathrm{Fe}(\mathrm{II})$ oxidation or iron-gallate ink complex from iron-gallic acid binding.

Inking was also performed on a fiber that was previously gelatin sized. Unexpectedly, very low amounts of gelatin were detected around the fiber, meaning that most of the gelatin was removed when the fiber was immersed in the ink. The penetration of iron inside the fiber was also much more limited than with the unsized fiber, showing that the former presence of a gelatin coating had delayed the migration of iron in the fiber during inking. This may be due to some chelation occurring between iron and gelatin that may prevent iron diffusion inside the fiber. Indeed gelatin bears carboxylate groups which can interact with iron(II) and iron(III).

To understand the impact of gelatin resizing of original documents during conservation treatments, gelatin was also sprayed on an inked fiber. Unexpectedly, the iron concentration inside this fiber was afterward largely reduced. A tentative explanation could be an entropic effect: during its application, gelatin remains outside the fiber, because it is made of macromolecules that are too large to penetrate the fiber structure. This is not the case of water that goes into the fiber and solubilises iron(II), provoking its release from the fiber. Iron is then chelated by gelatin that forms a gel during cooling, thus retaining iron. Finally, the concentration of iron inside the fiber is lowered, thus delaying the degradation of cellulose.

These observations should be taken with care as only a few samples could be analysed during this experiment, but nevertheless, it highlights the impact that gelatin can have on the iron gall ink corrosion, which is not only governed by chemical aspects, but also by physical behaviours.

\section{Materials and methods \\ Samples preparation \\ Choosing model fibers}

A paper was made from linen rags dating from the first half of the twentieth century at the Moulin du Verger paper mill (Puymoyen, France). The pulp used to make the sheets was prepared traditionally by stamper beating and contained no filler and no size. It is thus more representative of historical papers than the fibers used in the preliminary STXM testing [34], which consisted in bleached elemental chlorine free linen fibers coming from raw flax fibers.

Small pieces of the resulting dry sheet were damped with distilled water then individual fibers were sampled with a tweezer under a binocular magnifier. They were dried for one hour at $50 \%$ relative humidity $(\mathrm{RH})$ on a glass slide. A fiber was left untreated to be used as reference. Three other fibers were impregnated with the iron gallate ink and/or gelatin.

\section{Preparation of solutions and impregnation of fibers}

An iron-gallate ink solution was prepared using $40 \mathrm{~g} \cdot \mathrm{L}^{-1}$ of iron(II) sulfate $\left(\mathrm{FeSO}_{4} \cdot 7 \mathrm{H}_{2} 0\right.$, Sigma Aldrich) and $9 \mathrm{~g} \cdot \mathrm{L}^{-1}$ of gallic acid (monohydrate, Sigma). The solution was stirred for 3 days at ambient temperature to allow formation of iron-gallate ink precipitate. To avoid other sugar signatures than cellulosic fibers, this ink was made only from gallic acid and no gum was added. In addition, a gelatin solution was made at $4 \% \mathrm{w} / \mathrm{w}$ with a photographic grade gelatin (bovine source, Gelita, Restauration type 1, batch 073603, 269 Bloom, GMW, Germany). This relatively well characterized gelatin was already chosen in previous studies [25, 50]. It is also commonly used in conservation studios. The iron gallate ink solution and gelatin solution were used to impregnate three fibers:

- First an inked fiber, named "I". The fiber was immersed in the ink for $15 \mathrm{~s}$, then let for drying. As the resulting colour was very pale, the immersion was re-iterated, leading to a light blue aspect.

- Second, a sized and inked fiber, named "G_I. The sizing was done by immersing the fiber for $1 \mathrm{~min}$ in the warm gelatin solution $\left(50{ }^{\circ} \mathrm{C}\right)$. The fiber was then let 
to dry one day at $50 \% \mathrm{RH}$ and ambient temperature. It was afterward inked in a similar way as described above for the inked fiber I.

- Third, an inked fiber treated with gelatin named "I_G". The fiber was inked in a similar way as described above for the inked fiber I, then it was sprayed with the gelatin solution.

Due to synchrotron beam time constraint, G_I_G fiber was not considered in this study and a step-by-step approach was favoured with the above three mentioned fibers.

\section{Preparation of thin-sections}

STXM analysis requires the preparation of micro-slices thin enough to be transparent for C K-edge X-rays $(\sim 300 \mathrm{eV})$. To obtain these thin-sections of $100 \mathrm{~nm}$ thickness without any resin inclusion, fibers thin-sections were obtained with focussed ion beam (FIB). The thin sections were prepared at IEMN (Institut d'Electronique, de Microelectronique et de Nanotechnologies, Lille) using a FIB apparatus equipped with a SEM (scanning electron microscopy) imaging system (FEI corp STRATA DB 235). After the treatments described in the preceding section, the light blue fibers were deposited on Si wafers supports and dried at ambient temperature and $50 \%$ relative humidity $(\mathrm{RH})$ in closed glassware with preconditioned silica gel (Prosorb, 50\% RH).

Thin sections were prepared using the lift-out technique [51] (Additional file 1: Figure S1). The fiber was first coated with platinum by Ion Beam Induced deposition to protect the thin-section during cutting with the gallium ions beam. This layer and the Si wafer also aimed at preventing electrostatic charging of the samples during FIB milling. The thinsection was obtained in two steps: first, the fiber matter was removed from both part of the platinum deposit by sputtering with the gallium ions beam and secondly, the thin section was thinned down to $100 \mathrm{~nm}$ and was then tilted, allowing its final cutting and removal. The thin section was finally fixed on a post of a FIB lift-out grid having two wide posts in $\mathrm{V}$-shaped. SEM images of the thin-sections were obtained with an electron beam tension of $3 \mathrm{kV}$ in secondary electron mode. Between FIB milling and STXM analyses, the thinsections were stored in closed glass vessels with an oxygen absorber (RP, Long Life for Art, Germany) and humidity absorber (dry silica gel) allowing very low humidity level.

\section{Model compounds}

Model compounds are also necessary to obtain the NEXAFS reference signatures at each edge. Iron(II) chloride and iron(III) chloride, respectively named $\mathrm{Fe}^{\mathrm{II}}{ }_{-}$ref and $\mathrm{Fe}^{\mathrm{III}}{ }_{-}$ref, were used for the determination of the iron valence (Fe
L-edge) (spectra given by J. Wang, CLS). Indeed, iron sulfates were initially considered and although care had been taken to store them with oxygen absorbers during transportation, iron(II) sulfate was oxidized. The gelatin reference, named G, was extracted from the cross section of the I_G fiber. It corresponds to an excess of gelatin that was not in contact with the fiber. The gallic acid model compound, named Ac, corresponds to the deposition of a saturated ethanolic gallic acid solution on a TEM grid with a carbon mesh. The iron gall ink precipitate hereafter referred to as IGI was also prepared to identify the $\mathrm{C}$ K-edge signature specific of the precipitate. IGI was obtained by centrifugation of a solution containing iron(II) sulfate heptahydrate and gallic acid monohydrate with a molar ratio of 1:1. It was finely ground, put in suspension in ethanol and then deposited on a TEM grid with a carbon mesh.

\section{Scanning transmission X-ray microscopy (STXM) STXM mapping and NEXAFS spectroscopy}

Scanning transmission X-ray microscopy (STXM) is a technique that allows X-ray absorption imaging at the nanoscale. It requires synchrotron light. The beam is focused on the sample with a Fresnel zone-plate lens. Only the first order of the diffracted beam is selected with an order sorting aperture (OSA). The sample is scanned in $\mathrm{x}$ and $\mathrm{y}$ directions and for each energy. The microscope chamber is evacuated to 100 mtorr after sample insertion, then back-filled with helium. During energy scanning, an increase in the sample absorption is observed when the beam incident energy matches the electronic transition of chemical groups. The absorbance is also named optical density (O.D.) and is linked to the incident $\left(I_{0}\right)$ and transmitted $\left(I_{t}\right)$ intensities: O.D. $=-\ln \left(I_{t} / I_{0}\right)$.

A specific protocol was defined to analyse the samples as iron oxidation state was beam sensitive. First, the whole sample was mapped with low spatial resolution at energies close to Fe L-edges (700 eV, $707.8 \mathrm{eV}, 709.8 \mathrm{eV}$, and $730 \mathrm{eV}$ ). This approach made it possible to identify iron(II) and iron(III) rich areas that deserve a closer investigation while limiting sample irradiation. In these areas, stacks, consisting in a 3D data recording of sample absorption versus beam energy were then recorded (see Additional file 1: Figure S2) with a spatial resolution of $30 \mathrm{~nm}$. These stacks allow extraction of the NEXAFS spectra corresponding to a pixel, or to a specific region of several pixels. In this work, stacks were recorded from 700 to $720 \mathrm{eV}$ (Fe L-edge range), from 270 to $325 \mathrm{eV}$ (C K-edge range) and from 395 to $435 \mathrm{eV}$ (N K-edge range) (Additional file 1: Table S1). In order to limit iron reduction under the beam, Fe L-edge stacks were recorded first. Then the energy was shifted to the $\mathrm{C} \mathrm{K}$ - and/or N K-edges for complementary recording in the same areas. It made it possible to map on the same area the distribution of iron(II), iron(III), cellulose, gelatin and IGI compounds. 


\section{STXM data treatment}

The treatment of the stacks images was done using the aXis2000 software [52]. For each series of recording, the treatment consisted in (i) an alignment, (ii) a normalization with incident flux intensity to obtain absorption [optical density (O.D.)] maps, (iii) a subtraction of the images at characteristic energies from the image before the edge in order to enhance the component distribution visualization (see [53]), (iv) a stack fit to obtain the component maps (it performs a linear least squares fit of the data and fits the spectrum at each pixel with a sum of user-defined model spectra and a constant) and (v) a red green blue (RGB) treatment (to map the distribution of chosen reference compounds using colours). Spectra were extracted from stacks in several region of interest after the (ii) step. For the spectra comparison, an additional normalization to carbon or nitrogen quantities was done using the Athena software package [54].

\section{Supplementary Information}

The online version contains supplementary material available at https://doi.org/10. 1186/s40494-021-00593-2.

Additional file 1: Figure S1. Sample preparation protocol by Focussed lon beam (FIB). a inked fiber on a silicon wafer; $b$ Pt deposit to protect the fiber and avoid sample charging; $c$ trenches done with FIB (Ga ions beam) from each sides of the section; $d$ thin cross-section of the sample fiber, surrounded by $\mathrm{Pt}$ (above) and Si wafer (below); $d$ thin cross-sections on the A and C V-shape copper sample holder. Figure S2. STXM principle. Figure S3. Fiber I thin cross-section: a SEM observation, b and c STXM maps at the Fe L-edge. Map at $708 \mathrm{eV}$ (after subtraction of map at $700 \mathrm{eV}$ ) represent Fe" distributions; map at $709.8 \mathrm{eV}$ (after subtraction of map at $708 \mathrm{eV}$ ) represents Fe $\mathrm{III}^{\prime \prime}$ distributions. Arrow represents a region with a precipitate deposit at cross-section surface. Figure S4. Fiber G_I thin cross-section: a SEM observation, b and c STXM maps at the Fe L-edge. Map at $708 \mathrm{eV}$ (after subtraction of map at $700 \mathrm{eV}$ ) represent Fe" distributions; map at $709.8 \mathrm{eV}$ (after subtraction of map at $708 \mathrm{eV}$ ) represents Fell' distributions. Figure S5. NEXASF spectra of three different regions of fiber G_I at the N-K edge (see Fig. 4). Figure S6. Fiber I_G thin cross-section: a SEM observation, b STXM map at N K-edge: $401 \mathrm{eV}$ (subtracted by maps at $398 \mathrm{eV}$ ) representing gelatin distribution c and fSTXM at the C K-edge at $288.3 \mathrm{eV}$ and $286.7 \mathrm{eV}$ (from which maps at $280 \mathrm{eV}$ were subtracted), representing respectively gelatin and fiber major presence in bright. $d$ and e STXM maps at the Fe L-edge. Map at $708 \mathrm{eV}$ (after subtraction of map at $700 \mathrm{eV}$ ) represent Fe" distributions; map at $709.8 \mathrm{eV}$ (after subtraction of map at $708 \mathrm{eV}$ ) represents Fe'll distributions. Table S1. STXM NEXAFS measurements conditions.

\section{Acknowledgements}

We thank Mr Bréjoux from the Moulin du Verger papermill for preparing the rag paper used in this study. We also thank the Canadian Light Synchrotron for granting access to the beamline and Mr Wang for his help and fruitful discussion during experiments.

\section{Authors' contributions}

AG prepared the samples fibers. AG and VR conceived the experiments. All the authors took part in the STXM experiment. AG analyzed the data. AG and VR wrote the manuscript. PM and AM provided critical feedback and helped shape the research, analysis and manuscript. All authors read and approved the final manuscript.

\section{Funding}

This work was supported by French state funds managed by the ANR within the "Investissements d'Avenir" program (reference ANR-11-IDEX-0004-02) and more specifically within the framework of the Cluster of Excellence MATISSE.

\section{Availability of data and materials}

The datasets used and/or analyzed during the current study are available from the corresponding author on reasonable request.

\section{Declarations}

\section{Competing interests}

The authors have no relevant financial or non-financial interests to disclose.

\section{Author details}

${ }^{1}$ Centre de Recherche sur la Conservation des Collections (CRC, CNRS USR 3224), Sorbonne Université, Muséum National d'Histoire Naturelle, 36 rue Geoffroy St Hilaire, 75005 Paris, France. ${ }^{2}$ Laboratoire de Réactivité de Surface, Sorbonne Université, Campus UPMC, CNRS, UMR 7197, 4 place Jussieu, 75005 Paris, France.

Received: 7 May 2021 Accepted: 12 September 2021

Published online: 02 October 2021

\section{References}

1. Kolar J, Strilic M. Iron gall inks: on manufacture, characterisation, degradation and stabilisation. Ljubljana: National and University Library; 2006.

2. Teixeira N, Nabais P, de Freitas V, Lopes JA, Melo MJ. In-depth phenolic characterization of iron gall inks by deconstructing representative lberian recipes. Sci Rep. 2021;11:8811.

3. Díaz Hidalgo RJ, Córdoba R, Nabais P, Silva V, Melo MJ, Pina F, Teixeira N, Freitas $V$. New insights into iron-gall inks through the use of historically accurate reconstructions. Herit Sci. 2018;6:63.

4. Gimat A, Kasneryk V, Dupont A-L, Paris S, Averseng F, Fournier J, Massiani P, Rouchon V. Investigating the DMPO-formate spin trapping method for the study of paper iron gall ink corrosion. New J Chem. 2016;40:9098-110.

5. Gimat A, Dupont A-L, Lauron-Pernot H, Paris S, Rouchon V, Massiani P. Behavior of cellobiose in iron-containing solutions: towards a better understanding of the dominant mechanism of degradation of cellulosic paper by iron gall inks. Cellulose. 2017;24:5101-15.

6. Rouchon V, Belhadj O, Duranton M, Gimat A, Massiani P. Application of Arrhenius law to DP and zero-span tensile strength measurements performed on iron gall ink impregnated papers : relevance of artificial ageing protocols. Appl Phys A. 2016;122:1-10.

7. Neevel JG. Phytate: a potential conservation agent for the treatment of ink corrosion caused by iron gall inks. Restaurator. 1995;16:143-60.

8. Neevel JG. The development of a new conservation treatment for ink corrosion, based on the natural anti-oxidant phytate. Int J Forensic Doc Exam. 1999:5:130-7.

9. Messager M, Rouchon V. Damaged blue papers: optimising consolidation while preserving original colour. J Pap Conserv. 2013;14:9-19.

10. Kolar J, Mozir A, Strlic M, De Bruin G, Pihlar B, Steemers T. Stabilisation of iron gall ink: aqueous treatment with magnesium phytate. E-Preserv. 2007:4:19-24.

11. Rouchon V, Durocher B, Pellizzi E, Stordiau-Pallot J. The water sensitivity of iron gall ink and its risk assessment. Stud Conserv. 2009:54:236-54.

12. Liu Y, Cigić I, Strlič M. Kinetics of accelerated degradation of historic iron gall ink-containing paper. Polym Degrad Stab. 2017. https://doi.org/10.1016/j. polymdegradstab.2017.07.010.

13. Banik G, Brückle I. Paper and water: a guide for conservators. Amsterdam: Butterworth-Heinemann; 2011.

14. Yang L, Fogden A, Pauler N, Savborg O, Kruse B. A novel method for studying ink penetration of a print. Nord Pulp Pap Res J. 2005;20:423-9.

15. Kappel $\mathrm{CUH}$. Measurement of printing ink penetration in uncoated papers and is influence on print quality. Montreal: 94th Annual Meeting; 2008. p. B539-42.

16. Reissland B. Visible progress of paper degradation caused by iron gall inks. In: Iron Gall Ink Meeting. Newcastle upon Tyne: University of Northumbria; 2000. p. 67-72. 
17. LiY, He B. Characterization of ink pigment penetration and distribution related to surface topography of paper using confocal laser scanning microscopy. BioResources. 2011;6:2690-702.

18. Eriksen $\varnothing$, Gregersen $\varnothing W$. A study of ink pigment penetration and optical properties of laboratory sheets using scanning electron microscope. Trondheim: 2004 Progress in Paper Physics Seminar; 2004. p. 216-8.

19. Myllys M, Häkkänen H, Korppi-Tommola J, Backfolk K, Sirviö P,Timonen J. X-ray microtomography and laser ablation in the analysis of ink distribution in coated paper. J Appl Phys. 2015;117:144902.

20. Budnar M, Ursic M, Simcic J, Pelicon P, Kolar J, Selih VS, Strlic M. Analysis of iron gall inks by PIXE. Nucl Instrum Methods Phys Res Sect B-Beam Interact Mater At. 2006;243:407-16.

21. Eklund D, Salminen P. Water sorption in paper during short times. Appita J. 1987:40:340-6.

22. Garlick K. A brief review of the history of sizing and resizing practices. In: AIC Book Paper Group Annual, vol. 5. Washington: American Institute for Conservation; 1986. p. 94-107.

23. Waterhouse J, Barrett T. The aging characteristics of European handmade papers: 1400-1800. Tappi J. 1991;74:207-12.

24. Rouchon V, Pellizzi E, Janssens K. FTIR techniques applied to the detection of gelatine in paper artifacts: from macroscopic to microscopic approach. Appl Phys A. 2010;100:663-9.

25. Gimat A, Michelin A, Belhadj O, Pellizzi E, Massiani P, Rouchon V. Paper sizing with gelatine: from the macro- to the nano-scale. Cellulose. 2021. https://doi. org/10.1007/s10570-020-03655-Z.

26. Wu W, Li B, Hou H, Zhang H, Zhao X. Identification of iron-chelating peptides from Pacific cod skin gelatin and the possible binding mode. J Funct Foods. 2017;35:418-27.

27. Titus S, Schneller R, Huhsmann E, Hähner U, Banik G. Stabilising local areas of loss in iron gall ink copy documents from the Savigny estate. Restaurator. 2009:30:16-50.

28. Kolbe G. Gelatine in historical paper production and as inhibiting agent for iron-gall ink corrosion on paper. Restaurator. 2004;25:26-39.

29. Poggi G, Sistach M, Marin E, García J, Giorgi R, Baglioni P. Calcium hydroxide nanoparticles in hydroalcoholic gelatin solutions (GeolNan) for the deacidifcation and strengthening of papers containing iron gall ink. J Cult Herit. 2015. https:/doi.org/10.1016/j.culher.2015.10.005.

30. Proost $\mathrm{K}$, Janssens $\mathrm{K}$, Wagner B, Bulska E, Schreiner M. Determination of localized $\mathrm{Fe}^{2+} / \mathrm{Fe}^{3+}$ ratios in inks of historic documents by means of muXANES. Nucl Instrum Methods Phys Res Sect B-Beam Interact Mater At. 2004;213:723-8.

31. Arcon I, Kolar J, Kodre A, Hanzel D, Strlic M. XANES analysis of Fe valence in iron gall inks. X-Ray Spectrom. 2007;36:199-205.

32. Wilke M, Hahn O, Woodland AB, Rickers K. The oxidation state of iron determined by Fe K-edge XANES - application to iron gall ink in historical manuscripts. J Anal At Spectrom. 2009;24:1364-72.

33. Rouchon V, Duranton M, Burgaud C, Pellizzi E, Lavédrine B, Janssens K, de NolfW, Nuyts G, Vanmeert F, Hellemans K. Room-temperature study of iron gall ink impregnated paper degradation under various oxygen and humidity conditions: time-dependent monitoring by viscosity and X-ray absorption near-edge spectrometry measurements. Anal Chem. 2011;83:2589-97.

34. Rouchon V, Bernard S. Mapping iron gall ink penetration within paper fibres using scanning transmission X-ray microscopy. J Anal At Spectrom. 2015;30:635-41.

35. Hitchcock AP, Dynes JJ, Johansson G, Wang J, Botton G. Comparison of NEXAFS microscopy and TEM-EELS for studies of soft matter. Micron Oxf Engl 1993. 2008;39:741-8.

36. Kaznatcheev KV, Karunakaran Ch, Lanke UD, Urquhart SG, Obst M, Hitchcock AP. Soft X-ray spectromicroscopy beamline at the CLS: commissioning results. Nucl Instrum Methods Phys Res Sect Accel Spectrometers Detect Assoc Equip. 2007;582:96-9.

37. Liu H, Xia J, Nie Z, Ma Y, Ma C, Zheng L, Hong C, Zhao Y. Iron L-edge and sulfur K-edge XANES spectroscopy analysis of pyrite leached by Acidianus manzaensis. Trans Nonferrous Met Soc China. 2015;25:2407-14

38. Benzerara $K$, Yoon TH, Menguy N, TyliszczakT, Brown GE. Nanoscale environments associated with bioweathering of a Mg-Fe-pyroxene. Proc Natl Acad Sci USA. 2005;102:979-82.
39. Cody GD, Brandes J, Jacobsen C, Wirick S. Soft X-ray induced chemical modification of polysaccharides in vascular plant cell walls. J Electron Spectrosc Relat Phenom. 2009;170:57-64.

40. Solomon D, Lehmann J, Kinyangi J, Liang B, Heymann K, Dathe L, Hanley K. Carbon (1s) NEXAFS spectroscopy of biogeochemically relevant reference organic compounds. Soil Sci Soc Am J. 2009;73:1817-30.

41. Leinweber P, Kruse J, Walley FL, Gillespie A, Eckhardt K-U, Blyth RIR, RegierT. Nitrogen K-edge XANES —an overview of reference compounds used to identify unknown organic nitrogen in environmental samples. J Synchrotron Radiat. 2007;14:500-11.

42. Bourdelle F, Benzerara K, Beyssac O, Cosmidis J, NeuvilleGordon DREB Jr, Paineau E. Quantification of the ferric/ferrous iron ratio in silicates by scanning transmission X-ray microscopy at the Fe L2,3 edges. Contrib Mineral Petrol. 2013;166:423-34.

43. Ponce A, Brostoff LB, Gibbons SK, Zavalij P, Viragh C, Hooper J, Alnemrat S, Gaskell KJ, Eichhorn B. Elucidation of the Fe(III) gallate structure in historical iron gall ink. Anal Chem. 2016;88:5152-8.

44. Krekel C. The chemistry of historical iron gall inks. Int J Forensic Doc Exam. 1999;5:54-8.

45. Jacobi E, Reissland B, Phan Tan Luu C, Van Velzen B, Ligterink F. Rendering the invisible visible. J Pap Conserv. 2011;12:25-34.

46. Belhadj O, Phan Tan Luu C, Jacobi E, Meslet-Struyve S, Vez S, Reissland B, Rouchon V. The Dutch Fe-migration mending test: exploring further areas of use. $J$ Pap Conserv. 2014;15:9-15.

47. Smythe CV, Schmidt CLA. Studies on the mode of combination of iron with certain proteins, amino acids, and related compounds. J Biol Chem. 1930;88:241-69.

48. Qian M, Liu M, Eaton JW. Transition metals bind to glycated proteins forming redox active "glycochelates": implications for the pathogenesis of certain diabetic complications. Biochem Biophys Res Commun. 1998;250:385-9.

49. Helminger M, Wu B, Kollmann T, Benke D, Schwahn D, Pipich V, Faivre D, Zahn D, Cölfen H. Synthesis and characterization of gelatin-based magnetic hydrogels. Adv Funct Mater. 2014:24:3187-96.

50. Dupont A-L. Gelatine sizing of paper and its impact on the degradation of cellulose during ageing. Amsterdam: Universiteit van Amsterdam; 2003.

51. Giannuzzi LA, Stevie FA. A review of focused ion beam milling techniques for TEM specimen preparation. Micron. 1999;30:197-204.

52. Hitchcock AP. aXis2000-analysis of X-ray images and spectra. In: McMastre Univ. 2019. http://unicorn.mcmaster.ca/aXis2000.html. Accessed 29 Sep 2016.

53. Benzerara K, Yoon TH, Tyliszczak T, Constantz B, Spormann AM, Brown GE. Scanning transmission $X$-ray microscopy study of microbial calcification. Geobiology. 2004:2:249-59.

54. Ravel B, Newville M. ATHENA, ARTEMIS, HEPHAESTUS: data analysis for X-ray absorption spectroscopy using IFEFFIT. J Synchrotron Radiat. 2005;12:537-41.

\section{Publisher's Note}

Springer Nature remains neutral with regard to jurisdictional claims in published maps and institutional affiliations.

\section{Submit your manuscript to a SpringerOpen ${ }^{\circ}$ journal and benefit from:}

- Convenient online submission

- Rigorous peer review

- Open access: articles freely available online

- High visibility within the field

- Retaining the copyright to your article

Submit your next manuscript at $\boldsymbol{\nabla}$ springeropen.com 